\title{
Hair Loss after Varicella Zoster Virus Infection
}

\author{
Lara El Hayderi Nazli Nikkels-Tassoudji Arjen F. Nikkels \\ Department of Dermatology, CHU Sart Tilman, University of Liège, Liège, Belgium
}

\section{Key Words}

Varicella zoster virus · Alopecia $\cdot$ Hair follicle $\cdot$ Alopecia areata

\begin{abstract}
Varicella zoster virus (VZV) cutaneous infection occurs predominantly in epidermal and infundibular keratinocytes and accessorily in dermal dendritic cells. These latter cells play a role in cicatricial processes. Two patients are presented with localized alopecia after VZV infection. A 4-year-old girl presented localized hair loss affecting about $20 \%$ of her upper right eyelash immediately following the resolution of the varicella skin lesions. No regrowth was observed after 3 months. An 80-year-old woman with a prior history of localized alopecia areata of the left occipital area presented severe left herpes zoster affecting the V1 and V2 dermatomes. At precisely the same site of the previous episode, a localized plaque of alopecia areata recurred. After topical corticosteroid therapy, a progressive hair regrowth occurred after about 3 months. These case reports are the first relating cutaneous VZV infection as the origin for permanent cicatricial alopecia and transitory alopecia areata. Localized hair loss should be added to the cutaneous complications of VZV skin infection.
\end{abstract}

\section{Introduction}

The varicella zoster virus (VZV) is the responsible virus for chickenpox during childhood and herpes zoster (HZ) in elderly patients. A large array of cutaneous complications can be encountered during or following VZV skin infection [1]. Hyperpigmented or hypopigmented, slightly depressed, well delimited, small scars at the sites of previous skin lesions are the most frequent sequels of varicella. They sometimes resemble anetoderma. In contrast, cutaneous scarring following $\mathrm{HZ}$ is rare and only observed in cases with severe cutaneous lesions. The second most frequent cutaneous complication of varicella and HZ is a superinfection of the skin lesions by Staphylococcal bacteria [1]. 
As far as we know, permanent localized cicatricial hair loss following chickenpox and shingles-induced transitory alopecia areata (AA) has never been reported previously.

\section{Case Report}

Case 1

A 4-year-old girl without any particular medical, atopic or surgical history presented severe varicella with high fever and a large number of skin lesions, affecting the entire tegument. She presented a typical onset of chickenpox at the cephalic extremity. She did not take any medication and had not previously received the varicella vaccine. She also presented numerous facial lesions and in particular a lesion located on her upper right eyelid. An immunohistochemical staining with specific VZV gE antibodies on a Tzanck smear of a skin lesion of the thorax sustained the diagnosis of VZV skin infection [2]. Serology revealed positive VZV-specific IgM and IgG. As no atopic background or immunodeficiency was present, no antiviral treatment was initiated. Her mother administered supportive therapy using paracetamol and topical antiseptics. Spontaneous resolution of the skin lesions and the systemic accompanying signs occurred after about one week. Following resolution of the crusted right eyelash lesion, she presented focal skin atrophy with complete hair loss. There were no exclamation marks favoring AA. No concurrent scalp or eyebrow alopecic involvement was noted. Four weeks later, she still presented numerous small slightly erythematous and depressed varicella scars. After 2 months, there were no signs of eyelash hair regrowth and atrophy with partial border retraction of the eyelash was still present (fig. 1).

\section{Case 2}

An 80-year-old woman without any significant medical history presented at the emergency ward for a sudden appearance of severe headache affecting the left occipital site. There was no drug intake other than NSAIDs for joint pain. Clinical examination revealed well-limited erythematous, papulopustular, and some partially crusted lesions behind her left ear and the left occipital area, evoking HZ. Furthermore, she presented a well-restricted occipital plaque with total hair loss, resembling AA. An erythematovesicular lesion was present in the center of this alopecic plaque (fig. 2). This plaque appeared at the same time as the pain and the HZ eruption. No other alopecic lesions were noted on her scalp, face or body. The patient recalled having AA 20 years ago at precisely the same site, following the death of her husband. Due to the atypical presentation, a 3-mm punch biopsy was performed under local anesthesia of the lesion in the center of the alopecic plaque. Histopathologic examination revealed suprabasal acantholysis and necrotic of the epidermal and infundibular keratinocytes (fig. 3). A superficial and bulge-surrounding lymphomonocytic inflammatory infiltrate was present. Immunohistochemistry revealed the presence of the VZV-specific immediate early (IE)63 and late gE in epidermal and deep infundibular keratinocytes [2]. Herpes simplex virus (HSV)-I and -II antigens were not detected. Oral therapy with valaciclovir (Zelitrex ${ }^{\circledR}$, GSK, $3 \times 1,000 \mathrm{mg} /$ day, 7 days) was initiated. One week later, merely some small, crusted lesions were still observed, but the pain was still severe and debilitating. After one week gabapentin $(3 \times 100 \mathrm{mg} /$ day $)$ achieved a significant clinical relief of pain. This treatment was tapered after 2 months and then interrupted. No residual postherpetic neuralgia was observed. Topical very potent corticosteroids (clobetasol dipropionate lotion, $1 \times /$ day) were administered at the alopecic plaque. A progressive regrowth was observed with a complete hair regrowth after about 5 months. 
El Hayderi et al.: Hair Loss after Varicella Zoster Virus Infection

\section{Discussion}

During varicella and HZ skin lesions, active viral replication occurs in epidermal keratinocytes, causing intraepidermal acantholysis and vesiculation, clinically recognized as cutaneous vesicles $[3,4]$. Furthermore, VZV active replication and infection can also be observed in infundibular keratinocytes of the pilosebaceous structures. This can lead to VZV folliculitis that presents either as localized, dermatome restricted, or vesiculopustular folliculitis [5-12].

In contrast to HSV mucocutaneous infections, VZV also infects FXIIIa positive dermal dendritic cells $[2,7]$. These latter cells play a significant role in cutaneous healing as well as hypertrophic and keloid scarring processes [2]. The VZV-infected dermal dendrocytes present a non-cytopathic, low permissive viral infection $[2,7]$. This infection may lead to a dysfunction of the dermal dendrocytes and probably play a role in the development of varicella scars [2]. Hence, in the first case, the severe VZV blepharitis and concomitant VZV dermal dendrocyte infection may provide an explanation for the destruction of the pilosebaceous structures, probably by the VZV infection-elicited lymphohistiocytic infiltrate. The diagnosis of VZV-related permanent cicatricial hair loss is furthermore supported by the absence of eyelash hair regrowth after 3 months and the persistence of a slightly retracted, localized cutaneous atrophy at the site of a previous VZV lesion. The absence of other alopecic lesions, the cicatricial aspect of the eyelash, as well as the absence of hair regrowth and exclamation marks does not support a diagnosis of eyelash AA [13]. AA is one of the most frequent non-scarring cutaneous autoimmune diseases mediated by Th1 lymphocytes $[14,15]$. AA can be induced by a large variety of triggering factors, including stress, medication, etc. In the presented case, the pain during $\mathrm{HZ}$ could be considered as a stress factor, potentially triggering recurrent AA. Another possibility is that the HZ-accompanying cutaneous inflammatory reactions act as a Koebner phenomenon inducing AA [16].

HZ-induced AA and varicella-associated permanent cicatricial alopecia should be added to the cutaneous complications of VZV skin infection.

In conclusion, permanent cicatricial hair loss following chickenpox and shingles-induced transitory AA represent new complications of VZV cutaneous infections, as far as we are aware of, not previously reported in the literature.

\section{References}

1 El Hayderi L, Nikkels AF: Dermatological manifestations of varicella; in Lueng A (ed): Varicella. New York, NOVA publishers, 2013, in press.

2 Nikkels AF, Debrus S, Sadzot-Delvaux C, Piette J, Delvenne P, Rentier B, Piérard GE: Comparative immunohistochemical study of herpes simplex and varicella-zoster infections. Virchows Arch A Pathol Anat Histopathol 1993;422:121-126.

-3 Nikkels AF, Debrus S, Sadzot-Delvaux C, Piette J, Rentier B, Piérard GE: Localization of varicella-zoster virus nucleic acids and proteins in human skin. Neurology 1995;45(12 suppl 8):S47-S49.

-4 Arvin AM, Moffat JF, Sommer M, Oliver S, Che X, Vleck S, Zerboni L, Ku CC: Varicella-zoster virus T cell tropism and the pathogenesis of skin infection. Curr Top Microbiol Immunol 2010;342:189-209.

5 Walsh N, Boutilier R, Glasgow D, Shaffelburg M: Exclusive involvement of folliculosebaceous units by herpes: a reflection of early herpes zoster. Am J Dermatopathol 2005;27:189-194.

6 Muraki R, Iwasaki T, Sata T, Sato Y, Kurata T: Hair follicle involvement in herpes zoster: pathway of viral spread from ganglia to skin. Virchows Arch 1996;428:275-280.

7 Iwasaki T, Muraki R, Kasahara T, Sato Y, Sata T, Kurata T: Pathway of viral spread in herpes zoster: detection of the protein encoded by open reading frame 63 of varicella-zoster virus in biopsy specimens. Arch Virol Suppl 2001;17:109-119.

-8 Nikkels AF, Pierard GE: Hair follicle involvement in herpes zoster. Virchows Arch 1997;430:510-511.

9 Böer A, Herder N, Winter K, Falk T: Herpes folliculitis: clinical, histopathological, and molecular pathologic observations. Br J Dermatol 2006;154:743-746. 
El Hayderi et al.: Hair Loss after Varicella Zoster Virus Infection

10 Tilley DH, Satter EK, Kakimoto CV, Lederman ER: Disseminated verrucous varicella zoster with exclusive follicular involvement. Arch Dermatol 2012;148:405-407.

11 Weinberg JM, Mysliwiec A, Turiansky GW, Redfield R, James WD: Viral folliculitis. Atypical presentations of herpes simplex, herpes zoster, and molluscum contagiosum. Arch Dermatol 1997;133:983-986.

12 Al-Dhafiri SA, Molinari R: Herpetic folliculitis. J Cutan Med Surg 2002;6:19-22.

13 Modjtahedi BS, Kishan AU, Schwab IR, Jackson WB, Maibach HI: Eyelash alopecia areata: case series and literature review. Can J Ophthalmol 2012;47:333-338.

14 Gilhar A, Kalish RS: Alopecia areata: a tissue specific autoimmune disease of the hair follicle. Autoimmun Rev 2006;5:64-69.

15 Alkhalifah A, Alsantali A, Wang E, McElwee KJ, Shapiro J: Alopecia areata update: part I. Clinical picture, histopathology, and pathogenesis. J Am Acad Dermatol 2010;62:177-188.

16 Bellavista S, D'Antuono A, Gaspari V, Banzola N, Patrizi A: Acne keloidalis nuchae on herpes zoster scar in an HIV patient: isotopic response or not? G Ital Dermatol Venereol 2012;147:223-226.

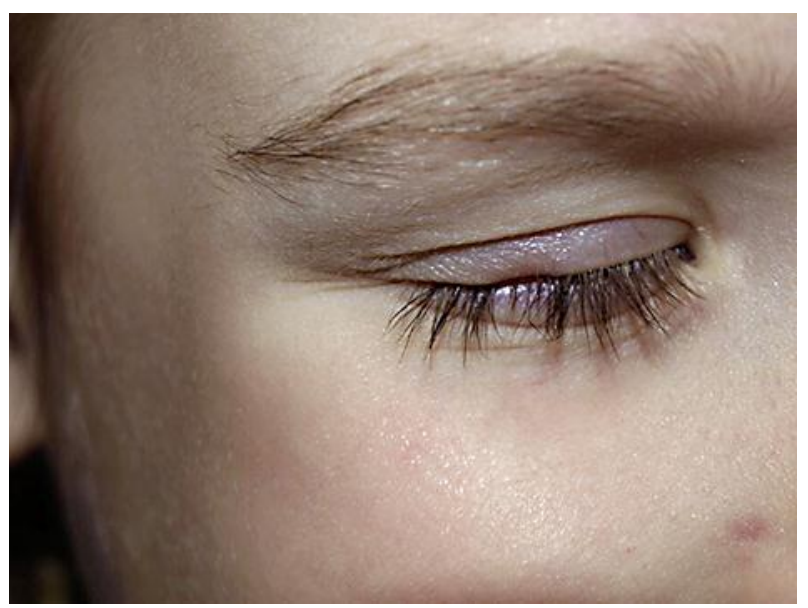

Fig. 1. Persisting upper right eyelash hair loss 3 months after the resolution of chickenpox. Note several cutaneous chickenpox scars.

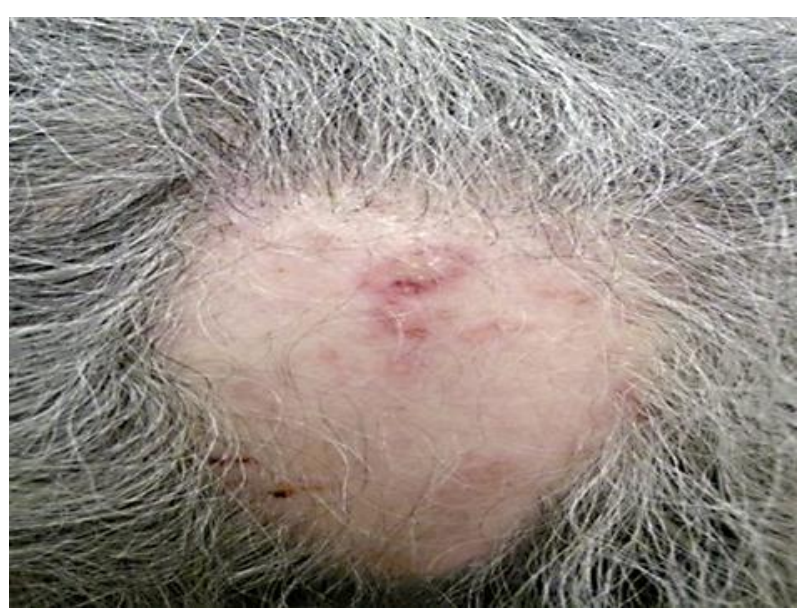

Fig. 2. Herpes zoster lesion in the center of an alopecia areata plaque. 


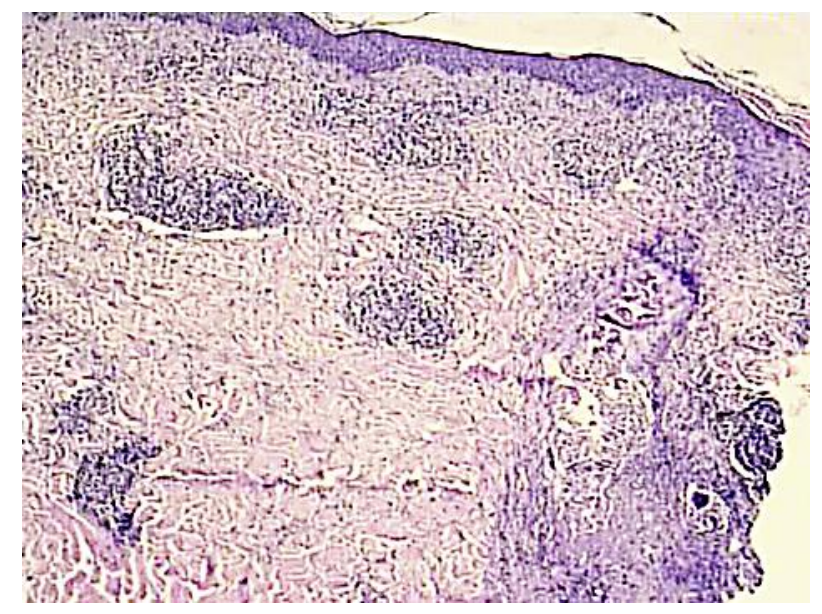

Fig. 3. VZV deep folliculitis $(\mathrm{HE}, \times 20)$. 